Research Article

\title{
Simulation and Analysis of Uncooled Microbolometer for Serial Readout Architecture
}

\author{
Musaed Alhussein and Syed Irtaza Haider \\ Computer Engineering Department, College of Computer and Information Sciences, King Saud University, \\ Riyadh 11451, Saudi Arabia
}

Correspondence should be addressed to Syed Irtaza Haider; sirtaza@ksu.edu.sa

Received 19 April 2016; Revised 3 July 2016; Accepted 11 July 2016

Academic Editor: Fanli Meng

Copyright (C) 2016 M. Alhussein and S. I. Haider. This is an open access article distributed under the Creative Commons Attribution License, which permits unrestricted use, distribution, and reproduction in any medium, provided the original work is properly cited.

\begin{abstract}
A detailed thermal behavior and theoretical analysis of uncooled resistive microbolometer is presented along with the proposed thermal imager simulator. An accurate model of a thermal detector is required to design a readout circuit that can compensate for the noise due to process variability and self-heating. This paper presents a realistic simulation model of microbolometer that addresses the fixed pattern noise, Johnson noise, and self-heating. Different simulations were performed to study the impact of infrared power and bias power on the performance of microbolometers. The microbolometers were biased with different bias currents along with different thermal parameters of the reference microbolometer to analyze the impact of self-heating on the thermal image. The proposed thermal imager simulator is used as a tool to visually analyze the impact of noise on the quality of a thermal image. This simulator not only helps in compensating the noise prior to the implementation in Analog Design Environment, but also can be used as a platform to explore different readout architectures. In this work, serial readout architecture was simulated with a row of blind microbolometers that served as a reference. Moreover, the algorithm for the proposed thermal imager simulator is presented.
\end{abstract}

\section{Introduction}

Over the last decade, uncooled thermal imagers have gained significant importance due to their low-cost and highperformance solution. These thermal imagers are widely used in many applications including monitoring of thermal volcanic activity, medical equipment, condition monitoring, electrical inspection, heat-loss inspection of buildings, and military surveillance equipment [1-3]. As the technology advances, building a low-cost, low-power, compact thermal imager is a key challenge that involves research in many areas. These areas include the performance evaluation of different thermal detectors, material selection based on the compatibility of a thermal detector with the complementary metal-oxide semiconductor (CMOS) process, ROIC design, noise removal techniques, digitization of analog signals, and image processing.

The two important aspects of a thermal imaging system are FPA and the ROIC. An FPA is a two-dimensional array of thermal detectors used for detecting infrared radiation [4]. The acquired signal is converted to a measurable electrical parameter and then passed to the ROIC. The primary function of the ROIC is to perform signal conversion and to send the data to the outputs. An ROIC should compensate for the noise sources and detectors self-heating during the biasing, background radiation, and significant variation in operating temperature [1]. The output of the ROIC is digitized by an analog-to-digital converter (ADC) and passed to the digital signal processing unit, which produces the final thermal image.

Infrared imaging using uncooled microbolometer detectors has been drawing much attention in recent years owing to its low cost and wide military and civilian applications. Uncooled microbolometer detectors operate at room temperature, which eliminates the need for cryogenic cooling [5]. High thermal isolation maximizes the sensitivity of the thermal detector $[6,7]$. The selected material should have a high temperature coefficient of resistance (TCR), good thermal isolation, and good compatibility with standard CMOS fabrication processes in a cost-efficient way. Some of the commonly used materials for the fabrication of thermal detectors are vanadium oxide, titanium, and amorphous silicon [8]. 
Microbolometer detector arrays are fabricated using the microelectromechanical systems (MEMS) technology [5, 9]. Due to the variability within the material and process, each fabricated microbolometer has a different nominal resistance value corresponding to the same infrared radiation that introduces the FPN in detector arrays $[1,2,10]$.

The FPN introduces nonuniformity in the thermal imager and needs to be addressed while designing the ROIC. The uniform reference target is the most common solution for nonuniformity correction (NUC) [11, 12]. The uniform reference target provides the known input that can be used to evaluate the output of the thermal detectors. The most commonly used methods that use a uniform target are onepoint NUC and two-point NUC. Both correction algorithms are based on the assumption that every detector is a linear function of the incident radiant flux. One-point NUC is based on the assumption that every detector has identical responsivity and that only the offset of each detector needs to be corrected, whereas the two-point correction algorithm corrects the offset and the responsivity [13]. In [1], individual calibration of current sources for biasing thermal detectors was proposed to reduce the nonuniformity. One of the common techniques is to use a blind microbolometer as a reference device along with a differential readout circuit [2]. Another method compensates for the FPN by connecting four microbolometer cells in parallel and averaging the resistances of the microbolometers [14].

When thermal detectors are exposed to infrared radiation, their temperature changes due to the incoming radiant flux. To monitor this change, the detectors need to be electrically biased. Electrical biasing generates Joule heating and causes a change in the resistance of the microbolometer. This heating effect is referred to as the self-heating of the microbolometer $[5,10]$. Although the bias duration is usually very small as compared to the thermal time constant of the microbolometer, it significantly increases the temperature of the microbolometer. The reason for this is that bias power is much higher than infrared power, thus resulting in a large temperature drift due to the self-heating [15]. Heat generated by self-heating cannot be quickly dissipated through thermal conduction to the substrate. Readout circuits are required to have a complex circuit design and a high dynamic range if self-heating is uncompensated. Thus, the self-heating must be compensated to improve the performance of the readout circuit and, eventually, of the thermal imaging system.

In the past, self-heating compensation has been explored extensively. Since bias power is directly proportional to bias duration, one simple method to compensate the self-heating is to bias the microbolometer for a very short period as compared to the thermal time constant $(\tau)$ of the microbolometer, that is, $\left(t_{\mathrm{BIAS}} \ll \tau\right)[16]$. Another technique to compensate the self-heating is to bias the microbolometer with the lowest practical value of the current. The bias current should not be low enough such that it reduces the responsivity of the microbolometer [17]. A common compensation technique is to use a row of blind microbolometers that serves as a reference microbolometer $[1,5,13,18]$. A blind microbolometer is identical to a normal microbolometer but is not affected by incoming incident radiation; thus, it is only subjected to self-heating. A Wheatstone bridge along with a differential amplifier reduces the Joule heating by suppressing the common mode signals [3]. Another self-heating compensation technique is to use a dummy microbolometer that has the same thermal mass as that of a normal microbolometer but has higher thermal conductivity and does not require infrared shielding $[18,19]$. The high thermal conductance of a dummy microbolometer detector reduces the thermal time constant to a very low value; thus, it can respond to selfheating immediately.

ROIC is an important building block of a thermal imager, and different ROIC architectures have advantages and disadvantages based on the amplification and integration methodology. The most common architectures are pixelwise, column-wise [20], and serial [16]. Pixel-wise readout improves the noise performance of a microbolometer by increasing the integration time up to the frame rate; thus, it is more suitable to minimize the Johnson noise [21]. This approach is used in high-speed thermal imaging. The disadvantage of pixel-wise readout is that it increases the complexity and cost of the design. Column-wise readout reduces the number of amplifiers and integrators working in parallel, thus serving as a good compromise between area and speed. Serial readout is a time-multiplexed architecture that performs the readout pixel by pixel. It requires only one amplifier and one $\mathrm{ADC}$, thus reducing the power consumption and complexity of the design at the expense of speed. Usually, there is a tradeoff between speed and power consumption. The power consumption of serial readout is $P_{D}+P_{A}+P_{I}$, where $P_{D}$, $P_{A}$, and $P_{I}$ represent the power consumption in detector, amplifier, and integrator, respectively. Similarly, the power consumption of column-wise readout is $Y \cdot\left(P_{D}+P_{A}+P_{I}\right)$, where $Y$ is the number of columns in a focal plane array.

A comprehensive tool for modeling of image noise in CMOS image sensors is presented in [22]. The tool provides realistic visual effects of noise on image quality. In [23], the phenomenon of self-heating due to the operation of the readout circuit is analyzed on UL03191 detector. SC7900VL thermographic camera was used to measure the thermodynamic behavior of UL03191 detector. In [24], a software application, SIST, is presented which simulates the effect of optical lens, detector, and image processing algorithms. The application allows determination of the optimum design of an imager. In [25], an image generator computer program named SIMTERM is presented. The program consists of four basic modules: the targets editor module, the observation conditions module, the camera module, and the visualization module. The targets editor module enables creation and editing of thermal targets. The observations conditions module calculates radiant signals emitted by the target and creates the input image. The camera module calculates distortions of the input target image due to camera imperfection. The visualization module creates final output thermal image. SIMTERM is a virtual thermal imager simulator that is used for the training of the ground thermal camera operators and for familiarizing them with the functionality of the thermal camera.

To summarize our contribution, we proposed a thermal imager simulator along with the detailed thermal behavior 
and theoretical analysis of uncooled resistive microbolometer. A realistic simulation model of microbolometer that addresses the fixed pattern noise, Johnson noise, and selfheating is presented. The model captures the behavior of microbolometer and helps in designing a readout circuit that compensates for the self-heating and fixed pattern noise. The proposed simulator can be used as a platform to explore different design options for the readout architecture. The simulator helps in understanding and compensating the noise sources prior to the implementation of readout circuit in Analog Design Environment. The current research focuses on the reference-based serial readout architecture. Different simulations were performed to study the impact of bias power and infrared power on the response of the microbolometer. A quantitative comparative analysis between serial and columnwise readout architectures is also presented. This paper is organized as follows. Section 2 explains the theoretical analysis and thermal behavior of uncooled resistive microbolometers. It also presents the algorithm for the proposed thermal imager simulator. Finally, Section 3 provides the simulation results and discussion.

\section{Methodology}

In this section, a general system architecture of ROIC is presented, along with the theoretical analysis and thermal behavior of uncooled microbolometers. The microbolometers were biased for different bias currents to understand the self-heating behavior. The temperature variation of the microbolometers during the entire frame period is presented when they were biased only once or multiple times within the frame time. In this study, a reference-pair serial readout architecture was explored in which the reference microbolometer was read out every time a new row was selected. The impact of self-heating due to multiple biasing of the reference microbolometer was studied by varying the thermal conductance of the reference microbolometer and keeping its thermal capacitance the same as that of the normal microbolometer.

2.1. Theoretical Analysis of Uncooled Microbolometer. The analysis of uncooled microbolometer begins by solving the heat balance equation that can be expressed as [25]

$$
H \frac{d \Delta T}{d t}+G \Delta T=P_{\mathrm{BIAS}}+P_{\mathrm{IR}}
$$

where $P_{\text {BIAS }}$ is the self-heating power, $P_{\mathrm{IR}}$ is the infrared input power, $G$ is the thermal conductance, and $H$ is the thermal capacitance of uncooled microbolometer. The time response and the thermal behavior of the microbolometer are determined by the thermal time constant, thermal capacitance, and thermal conductance. The relationship among these three thermal parameters is defined as

$$
\tau=\frac{H}{G} .
$$

When the bias duration is very small as compared to the thermal time constant of microbolometer, that is, $\left(t_{\text {BIAS }} \ll \tau\right)$, then the resistance $R_{B}$ has linear dependence on the temperature and can be expressed as

$$
R_{B}\left(t_{\mathrm{BIAS}}\right)=R_{0}\left(1+\alpha \Delta T\left(t_{\mathrm{BIAS}}\right)\right)
$$

where $R_{B}\left(t_{\mathrm{BIAS}}\right)$ is the resistance of the microbolometer due to the self-heating, $t_{\mathrm{BIAS}}$ is the bias duration, $R_{0}$ is the nominal resistance of the microbolometer, $\alpha$ is the temperature coefficient of the resistance (TCR), and $\Delta T\left(t_{\text {BIAS }}\right)$ is the temperature change due to the pulse biasing of the microbolometer and is estimated as

$$
\left.\Delta T\left(t_{\mathrm{BIAS}}\right)\right|_{P_{\mathrm{IR}}=0}=\frac{P_{\mathrm{BIAS}}\left(t_{\mathrm{BIAS}}\right)}{H} t_{\mathrm{BIAS}} .
$$

If $I_{\text {BIAS }}$ is the constant bias current applied to the microbolometer during the readout, then the self-heating power can be expressed as follows:

$$
\begin{aligned}
P_{\mathrm{BIAS}}\left(t_{\mathrm{BIAS}}\right) & =I_{\mathrm{BIAS}}^{2} R_{B}\left(t_{\mathrm{BIAS}}\right) \\
& =I_{\mathrm{BIAS}}^{2} R_{0}\left(1+\alpha \Delta T\left(t_{\mathrm{BIAS}}\right)\right) \\
& =I_{\mathrm{BIAS}}^{2} R_{0}\left(1+\alpha\left(\frac{P_{\mathrm{BIAS}}\left(t_{\mathrm{BIAS}}\right)}{H} t_{\mathrm{BIAS}}\right)\right) .
\end{aligned}
$$

By solving the above equation for $P_{\mathrm{BIAS}}$, the self-heating power can be expressed as

$$
P_{\mathrm{BIAS}}\left(t_{\mathrm{BIAS}}\right)=\frac{I_{\mathrm{BIAS}}^{2} R_{0}}{1-I_{\mathrm{BIAS}}^{2} R_{0} \alpha\left(t_{\mathrm{BIAS}} / H\right)} .
$$

In order to accurately model the microbolometer, it is important to consider the Johnson noise for the resistive uncooled microbolometer. This noise is independent of the microbolometers material composition and exists even at zero bias. The Johnson noise rms voltage can be expressed as

$$
V_{n}=\sqrt{4 k T B R_{\mathrm{PIXEL}}}
$$

where $k$ is the Boltzmann constant, $T$ is the temperature in degrees kelvin, $B$ is the bandwidth, and $R_{\text {PIXEL }}$ is the resistance of the microbolometer at ambient temperature. When the focal plane array is exposed to the incident infrared power, the difference in radiant flux incoming on the microbolometer can be estimated as

$$
\Delta \phi_{\mathrm{IR}}=\frac{A_{b} \Delta T_{\text {scene }}}{4 F^{2}}\left(\frac{d P}{d T}\right)_{300 \mathrm{~K}, \Delta \lambda},
$$

where $\Delta T_{\text {scene }}$ is the difference between the scene and the ambient temperature and $(d P / d T)_{300 \mathrm{~K}, \Delta \lambda}$ is the temperature contrast in the infrared wavelength at an ambient temperature. $A_{b}$ is the area of the microbolometer pixel and $F$ is the optics number. The incoming radiant flux changes the temperature of the microbolometer and is given by

$$
\Delta T_{\mathrm{IR}}=\frac{\Delta \phi_{\mathrm{IR}} \beta \phi_{\Delta \lambda} \varepsilon_{\Delta \lambda}}{G}
$$


where $\beta$ is the fill factor of microbolometer, $\phi_{\Delta \lambda}$ is the transmission of optics, and $\varepsilon_{\Delta \lambda}$ is the absorption of microbolometer membrane in the infrared wavelength. When the bias power is zero, the microbolometer cools down based on the following expression:

$$
\Delta T_{\mathrm{COOL}}=T_{B}\left(e^{-(t / \tau)}\right)
$$

where $T_{B}$ is the temperature of the microbolometer at the end of the readout pulse, $t$ is the time between the end of the first pulse and the start of the second pulse for the reference microbolometer, and $\tau$ is the thermal time constant of the microbolometer.

2.2. ROIC Architecture. Figure 1 shows the general system architecture of the ROIC. The FPA consists of $X * Y$ normal microbolometers and a row of blind microbolometers that served as a reference microbolometer. The blind microbolometers were coated with a reflective layer to shield them from input incident infrared power. In the case of serial readout architecture, a current source with two outputs is desirable. The current source should be capable of providing a constant current throughout the readout time to all the microbolometers irrespective of their resistance variation due to the self-heating or process variability.

Each microbolometer, whether blind or normal, was connected to a current source through a P-type metal-oxidesemiconductor (PMOS) switch. The gate of the PMOS switch was controlled through the digital circuitry that generates the pulse sequence based on the readout topology. A differential approach was used at the preamplification stage, and, finally, digitization of the readout signal was performed using an ADC. The number of amplifiers and ADCs depends on the readout architecture. Thus, if the reference-based serial architecture was used, then only one amplifier and one ADC were used, as it is a time-multiplexed readout architecture.

2.3. Thermal Behavior of the Uncooled Microbolometer. It is very important to understand the overall thermal behavior of an infrared detector before designing the readout circuit. The thermal behavior of a microbolometer not only explains the self-heating response but also helps in choosing the right bias parameters. Each pixel in an FPA represents an individual microbolometer, and these pixels are addressed based on the readout topology. Figure 2 shows the temperature and resistance variation of the microbolometer due to the self-heating when the bias current was swept to $50 \mu \mathrm{A}$ for a bias duration of $6 \mu \mathrm{s}, 20 \mu \mathrm{s}$, and $50 \mu \mathrm{s}$. It can be seen from the figure that, for a bias duration of $50 \mu \mathrm{s}$, when the bias current was swept to $50 \mu \mathrm{A}$, the resistance of the microbolometer decreased by approximately $40 \mathrm{k} \Omega$ from its nominal value, which was equivalent to approximately $17 \mathrm{~K}$ rise in temperature due to the self-heating. Table 1 shows the thermal parameters of the microbolometer that were used for the simulation [4].

The bias current is one of the most critical parameters in ROIC design that if chosen appropriately reduces the impact of self-heating without introducing any complexity in the readout design. There is a direction relation between bias current and bias power. If the bias current is large, it will result in large self-heating of the microbolometer. Choosing an optimum bias current is a tricky task. The bias current should not be too small that the responsivity of the microbolometer is compromised, nor should it be too large that the temperature rise due to the self-heating is too large. We have presented a detailed analysis to determine the optimum bias parameters later in this section.

In case of the reference-pair serial readout, the applied bias current should be for a short duration so that each reference microbolometer has time to return to the stabilization temperature before the second pulse is applied to the same microbolometer within the frame time. Otherwise, the temperature would gradually increase to levels leading to overheating. So in order to prevent thermal runaway and to limit the detector noise, it is recommended to bias the microbolometers for a short duration.

Figure 3 shows the simulation result of microbolometer temperature variation in the absence of infrared power. Figure 3(a) shows the temperature variation of normal microbolometer since it was biased only once during the entire frame time, whereas Figure 3(b) shows the temperature variation of the reference microbolometer as it was biased every time when the new row in the FPA is selected for the readout. The incident infrared power was set to zero to observe the microbolometer temperature response due only to the self-heating. It can be seen from Figure 3(a) that the microbolometer had a nominal temperature before the readout. The nominal temperature is defined as the temperature difference of the microbolometer with the ambient temperature. This temperature could be either zero, when the incident infrared power was zero, or DC offset of several millikelvins when the microbolometers were exposed to the infrared radiation. The temperature of the microbolometer rises during the readout due to the bias power that resulted in self-heating of the microbolometer. The slope represents the temperature rise during the readout due to the selfheating, and it is linear when the bias duration is very small as compared to the thermal time constant of the microbolometer. At the end of the readout, the microbolometer temperature rises by approximately $600 \mathrm{mK}$. After the readout, the microbolometer cools down for the rest of the frame period.

Table 2 shows the pulse sequence for a pair-wise serial ROIC in which a pair of microbolometers were selected and biased during each pulse duration. The reference microbolometers were biased only once whereas the normal microbolometers were biased twice during the entire frame time. Once the readout of the selected pair was finished, the bias current source was switched to the next pair of microbolometers. Recently biased detector pair was left to cool off until the next pulse within the same frame time arrived. The reference microbolometer cooled down for the rest of the frame time.

Table 3 shows the pulse sequence for the referencepair serial readout. In Figure 3(b), the microbolometer was biased $X$ number of times during the entire frame period, where $X$ is the number of rows in the FPA. The reference microbolometer needed to be addressed every time a new row was selected. This reference microbolometer could either 


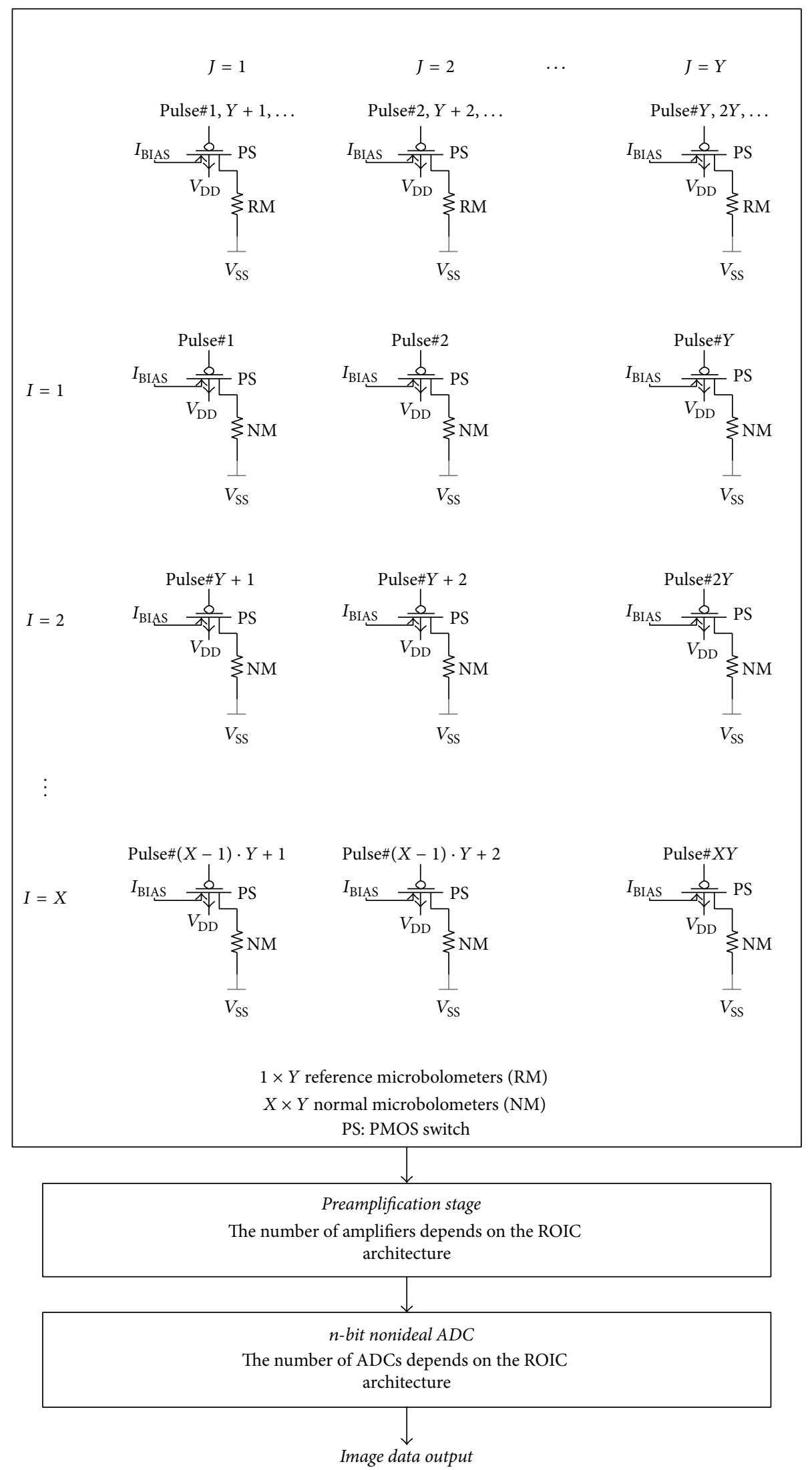

FIGURE 1: General system architecture of the ROIC, where the pulse sequence is based on the reference-pair serial readout as mentioned in Table 3. 




Figure 2: Microbolometer thermal behavior: bias current $(x-$ axis) versus resistance and temperature ( $y$-axis) for various bias durations.

have the same thermal parameters as those of the normal microbolometer or have different thermal conductance to improve the thermal behavior. It can be seen from Figure 3(b) that, because of the multiple readouts, the temperature of the reference microbolometer rises very high due to the self-heating. A steady-state behavior was expected when the microbolometer attained saturation of the temperature difference. As can be seen in the figure, the temperature of the reference microbolometer rises by approximately $6.5 \mathrm{~K}$ at around $50 \mathrm{~ms}$ and then oscillated within $500 \mathrm{mK}$ until the end of the frame period.

The reference microbolometer experienced a large temperature drift due to the self-heating as it was biased every time a new row was selected. To reduce the self-heating, one can use a method that increases the thermal conductance of the reference microbolometer, whereas the thermal capacitance remains the same as that of the normal microbolometer. Figure 4 shows the pulse sequence for the first reference microbolometer RM $(1,1)$ and rowl normal microbolometers $\mathrm{NM}(1, j)$ of FPA, where the reference microbolometers were biased with a current of $I_{\text {BIAS }}$ for a short duration of $T_{\text {BIAS }}$, separated by a relatively long interval $T_{\mathrm{COOL}}$.

If $T_{\text {BIAS }}$ was $6 \mu$ s and FPA consists of $128 \times 92$ pixels, then $T_{\mathrm{COOL}}$ for each reference microbolometer would be $T_{\mathrm{COOL}}=$ $6 \mu \mathrm{s} \times 91=546 \mu \mathrm{s}$. During $T_{\mathrm{COOL}}$ time, the microbolometer cooled down based on (10). The simulation was performed in which the reference microbolometers with thermal conductance values of $9.5 \mathrm{nW} \cdot \mathrm{K}^{-1}, 34 \mathrm{nW} \cdot \mathrm{K}^{-1}$, and $144 \mathrm{nW} \cdot \mathrm{K}^{-1}$ were biased with $10 \mu \mathrm{A}$ and $20 \mu \mathrm{A}$ for a bias duration of $6 \mu \mathrm{s}$, $20 \mu \mathrm{s}$, and $250 \mu \mathrm{s}$. Table 4 shows the temperature increase of reference microbolometer in one pulse $T_{B}$ and before the start of the second pulse $\Delta T_{\mathrm{COOL}}$ using (4) and (10), respectively. Table 4 helps in determining the optimum bias parameters for the microbolometer.

As seen in Table 4 , if the bias duration was $250 \mu$ s, then the temperature increase due to the self-heating in just one single
TABLE 1: Thermal parameters of the microbolometer.

\begin{tabular}{lc}
\hline Parameter & Value \\
\hline Microbolometer pixel area, $A_{b}\left(\mathrm{~m}^{2}\right)$ & $6.25 e-10$ \\
Microbolometer fill factor, $\beta(\%)$ & 62 \\
Microbolometer absorption coefficient, $\varepsilon_{\Delta \lambda}(\%)$ & 92 \\
Transmission of infrared optics, $\phi_{\Delta \lambda}(\%)$ & 98 \\
Temperature contrast $(d P / d T)_{300 \mathrm{~K}, \Delta \lambda}\left(\mathrm{W} / \mathrm{K} \mathrm{m}^{2}\right)$ & 2.624 \\
Thermal conductance, $G(\mathrm{~W} / \mathrm{K})$ & $3.7 e-8$ \\
Thermal capacitance, $H(\mathrm{~J} / \mathrm{K})$ & $4.34 e-10$ \\
Temperature coefficient of resistance, $\alpha(\% / \mathrm{K})$ & -2.6 \\
Thermal time constant, $\tau(\mathrm{ms})$ & 11.7 \\
\hline
\end{tabular}

TABLE 2: Pulse sequence for a pair-wise serial ROIC.

\begin{tabular}{lc}
\hline Pulse \# & Selected microbolometer detector pair \\
\hline 1 & $\mathrm{RM}(1,1)$ and $\mathrm{NM}(1,1)$ \\
2 & $\mathrm{NM}(2,1)$ and $\mathrm{NM}(3,1)$ \\
3 & $\mathrm{NM}(4,1)$ and $\mathrm{NM}(5,1)$ \\
$\vdots$ & $\vdots$ \\
$X / 2$ & $\mathrm{NM}(X-1,1)$ and $\mathrm{NM}(X, 1)$ \\
$(X / 2)+1$ & $\mathrm{NM}(1,1)$ and NM $(2,1)$ \\
$(X / 2)+2$ & $\mathrm{NM}(3,1)$ and NM $(4,1)$ \\
$\vdots$ & $\quad \vdots$ \\
$X-1$ & $\mathrm{NM}(X-2,1)$ and $\mathrm{NM}(X-1,1)$ \\
$X$ & $\mathrm{RM}(1,2)$ and $\mathrm{NM}(1,2)$ \\
$\vdots$ & $\vdots$ \\
\hline
\end{tabular}

$X$ is the number of rows; $Y$ is the number of columns in the FPA; RM: reference microbolometer; NM: normal microbolometer.

TABLE 3: Pulse sequence for a reference-pair serial ROIC.

\begin{tabular}{lc}
\hline Pulse \# & Selected microbolometer detector pair \\
\hline 1 & $\mathrm{RM}(1,1)$ and $\mathrm{NM}(1,1)$ \\
2 & $\mathrm{RM}(1,2)$ and $\mathrm{NM}(1,2)$ \\
$\vdots$ & $\vdots$ \\
$Y$ & $\mathrm{RM}(1, Y)$ and $\mathrm{NM}(1, Y)$ \\
$Y+1$ & $\mathrm{RM}(1,1)$ and $\mathrm{NM}(2,1)$ \\
$Y+2$ & $\mathrm{RM}(1,2)$ and $\mathrm{NM}(2,2)$ \\
$\vdots$ & $\vdots$ \\
$2 Y$ & $\mathrm{RM}(1, Y)$ and $\mathrm{NM}(2, Y)$ \\
$\vdots$ & $\vdots$ \\
$X Y$ & $\mathrm{RM}(1, Y)$ and NM $(X, Y)$ \\
\hline
\end{tabular}

$X$ is the number of rows; $Y$ is the number of columns in the FPA; RM: reference microbolometer; NM: normal microbolometer.

pulse was very high. Since the reference microbolometer was biased every time when a new row in the FPA was selected, the temperature of the microbolometer would increase to levels leading to overheating. If the optimum value of bias current was applied for that long, then it will eventually result in thermal runaway. The bias parameters that should not be used to bias the reference microbolometer are marked with " $\dagger$ " in Table 4 , in order to prevent the thermal runaway. 


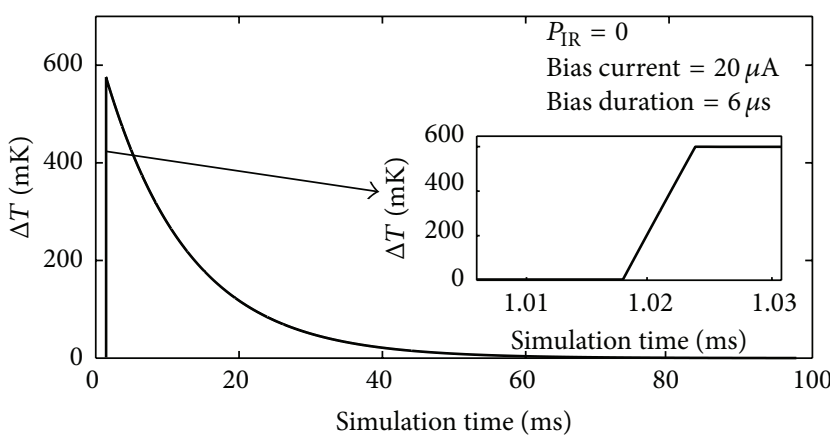

(a)

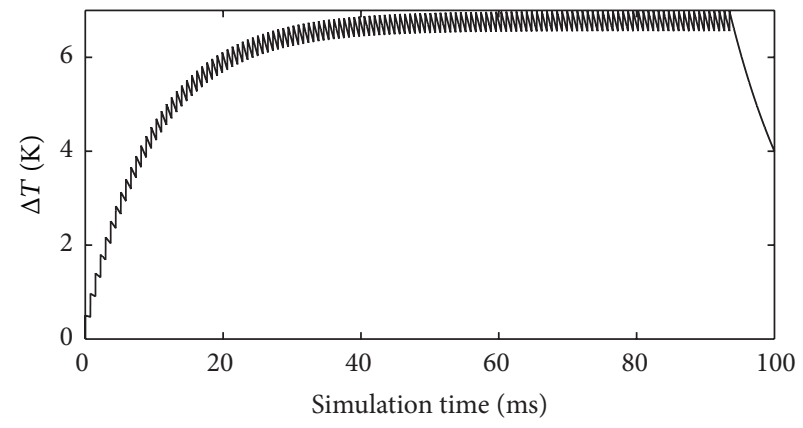

(b)

FIGURE 3: Temperature variation of the microbolometer, (a) single pulse and (b) multiple pulses, when the incident power was zero.

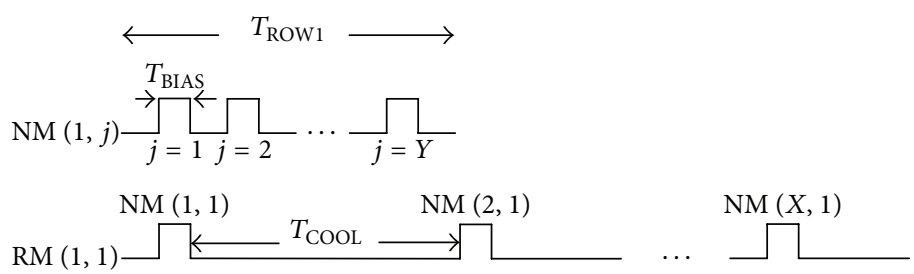

FIGURE 4: Pulse sequence for the first reference microbolometer RM $(1,1)$ and row1 normal microbolometers NM $(1, j)$ of FPA using referencepair serial readout.

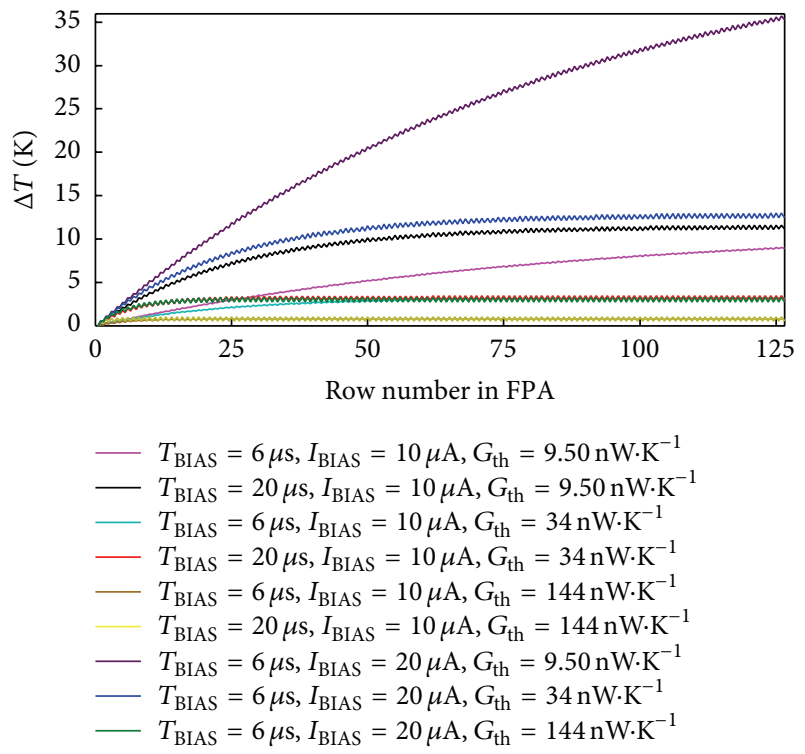

FIGURE 5: Temperature variation of the reference microbolometer for different bias parameters and thermal parameters.

Figure 5 shows the temperature variation of the reference microbolometer when it was biased multiple times during the frame period, for the parameters not marked with " $\dagger$ " in Table 4. If we consider that the acceptable temperature rise due to the self-heating is $4 \mathrm{~K}$ above the nominal temperature of the microbolometer, then, from Figure 5, the reference microbolometer could be biased with the parameters marked with “ $\ddagger$ ” in Table 4. The ROI was highlighted in Figure 2 where $I_{\mathrm{MIN}}$ was $10 \mu \mathrm{A}$ and $I_{\mathrm{MAX}}$ was $20 \mu \mathrm{A}$. From Table 4 , if the thermal conductance of the reference microbolometer was significantly larger than the thermal conductance of the normal microbolometers, then $I_{\mathrm{BIAS}}=I_{\mathrm{MAX}}$, whereas if the thermal conductance values of the reference and the normal microbolometers were the same, then $I_{\mathrm{BIAS}}=I_{\mathrm{MIN}}$. The optimum bias parameters will be $I_{\text {BIAS }}=20 \mu \mathrm{A}$ and $T_{\text {BIAS }}$ $=6 \mu \mathrm{s}$ for $G_{\mathrm{th}}=144 \mathrm{n} \cdot \mathrm{W} \cdot \mathrm{K}^{-1}$ whereas $I_{\text {BIAS }}=10 \mu \mathrm{A}$ and $T_{\text {BIAS }}$ $=6 \mu \mathrm{s}$ for $G_{\mathrm{th}}=34 \mathrm{n} \cdot \mathrm{W} \cdot \mathrm{K}^{-1}$.

The bias duration should be very small as compared to the thermal time constant of the reference microbolometer; otherwise, the temperature rise due to the self-heating during the readout would be nonlinear. If the microbolometer is biased for a longer duration, comparable to the thermal time constant of the microbolometer, then, for an optimum value of the bias current, the resistance of the microbolometer changes significantly and nonlinearly during the pulse bias period. This resistance change would be in the order of several kiloohms. Since the bias duration is too large, the voltage change over the period would be significant and nonlinear instead of negative ramp. It can cause a reduction in the dynamic range, that is, voltage range due to the significant voltage change over the period, thereby using up allowable voltage swing. Ideally, the output voltage of the amplifier would remain constant over temperature. However, due to the self-heating effects, the output voltage decreases with increasing temperature. In order to make sure that an amplified signal remains constant over temperature and well within the dynamic range of the readout circuitry, the microbolometer needs to be biased for a shorter duration.

The thermal conductance of a microbolometer depends on the thermal conductivity of the material and the design 
TABLE 4: Temperature increase of reference microbolometer for various bias parameters and thermal parameters.

\begin{tabular}{|c|c|c|c|c|c|c|}
\hline $\begin{array}{l}\text { Bias current, } \\
I_{\text {BIAS }}(\mu \mathrm{A})\end{array}$ & $\begin{array}{c}\text { Bias } \\
\text { duration, } \\
t_{\text {BIAS }}(\mu \mathrm{s})\end{array}$ & $\begin{array}{c}\text { Thermal } \\
\text { conductance, } \\
G_{\text {th }} \\
\left(\mathrm{nW} \cdot \mathrm{K}^{-1}\right)\end{array}$ & $\begin{array}{l}\text { Thermal time } \\
\text { constant, } \tau \\
\text { (ms) }\end{array}$ & $\begin{array}{c}\text { Temperature } \\
\text { increase in one } \\
\text { pulse, } T_{B}\end{array}$ & $\begin{array}{l}\text { Temperature before } \\
\text { the start of the second } \\
\text { pulse, } \Delta T_{\mathrm{COOL}}\end{array}$ & $\begin{array}{c}\text { Temperature at the } \\
\text { end of the second } \\
\text { pulse, } \\
T_{B}+\Delta T_{\mathrm{COOL}}\end{array}$ \\
\hline \multirow{9}{*}{10} & \multirow{3}{*}{6} & 9.50 & 45.68 & \multirow{3}{*}{$137.75 \mathrm{mK}$} & $136.11 \mathrm{mK}$ & $273.86 \mathrm{mK}$ \\
\hline & & $34.0^{\ddagger}$ & $12.76^{\ddagger}$ & & $131.98 \mathrm{mK}^{\ddagger}$ & $269.73 \mathrm{mK}^{\ddagger}$ \\
\hline & & $144^{\ddagger}$ & $3.01^{*}$ & & $114.89 \mathrm{mK}^{\ddagger}$ & $252.64 \mathrm{mK}^{\ddagger}$ \\
\hline & \multirow{3}{*}{20} & 9.50 & 45.68 & \multirow{3}{*}{$455.35 \mathrm{mK}$} & $437.56 \mathrm{mK}$ & $892.91 \mathrm{mK}$ \\
\hline & & $34.0^{\ddagger}$ & $12.76^{\ddagger}$ & & $394.82 \mathrm{mK}^{\ddagger}$ & $850.17 \mathrm{mK}^{\ddagger}$ \\
\hline & & $144^{\ddagger}$ & $3.01^{\ddagger}$ & & $248.74 \mathrm{mK}^{\ddagger}$ & $704.09 \mathrm{mK}^{\ddagger}$ \\
\hline & \multirow{3}{*}{$250^{\dagger}$} & $9.50^{\dagger}$ & $45.68^{\dagger}$ & \multirow{3}{*}{$5.01 \mathrm{~K}^{\dagger}$} & $3.04 \mathrm{~K}^{\dagger}$ & $8.05 \mathbf{K}^{\dagger}$ \\
\hline & & $34.0^{\dagger}$ & $12.76^{\dagger}$ & & $842.18 \mathrm{mK}^{\dagger}$ & $5.85 \mathrm{~K}^{\dagger}$ \\
\hline & & $144^{\dagger}$ & $3.01^{\dagger}$ & & $2.61 \mathrm{mK}^{\dagger}$ & $5.012 \mathrm{~K}^{\dagger}$ \\
\hline \multirow{9}{*}{20} & \multirow{3}{*}{6} & 9.50 & 45.68 & \multirow{3}{*}{$545.16 \mathrm{mK}$} & $538.61 \mathrm{mK}$ & $1.08 \mathrm{~K}$ \\
\hline & & 34.0 & 12.76 & & $522.26 \mathrm{mK}$ & $1.07 \mathrm{~K}$ \\
\hline & & $144^{\ddagger}$ & $3.01^{\ddagger}$ & & $454.66 \mathrm{mK}^{\ddagger}$ & $999.82 \mathrm{mK}^{\ddagger}$ \\
\hline & \multirow{3}{*}{$20^{\dagger}$} & $9.50^{\dagger}$ & $45.68^{\dagger}$ & \multirow{3}{*}{$1.759 \mathrm{~K}^{\dagger}$} & $1.69 \mathrm{~K}^{\dagger}$ & $3.45 \mathrm{~K}^{\dagger}$ \\
\hline & & $34.0^{\dagger}$ & $12.76^{\dagger}$ & & $1.53 \mathrm{~K}^{\dagger}$ & $3.29 \mathrm{~K}^{\dagger}$ \\
\hline & & $144^{\dagger}$ & $3.01^{\dagger}$ & & $1.47 \mathrm{~K}^{\dagger}$ & $3.23 \mathrm{~K}^{\dagger}$ \\
\hline & \multirow{3}{*}{$\mathbf{2 5 0 ^ { \dagger }}$} & $9.50^{\dagger}$ & $45.68^{\dagger}$ & \multirow{3}{*}{$14.41 \mathrm{~K}^{\dagger}$} & $8.75 \mathrm{~K}^{\dagger}$ & $23.16 \mathrm{~K}^{\dagger}$ \\
\hline & & $34.0^{\dagger}$ & $12.76^{\dagger}$ & & $2.42 \mathrm{~K}^{\dagger}$ & $16.83 \mathrm{~K}^{\dagger}$ \\
\hline & & $144^{\dagger}$ & $3.01^{\dagger}$ & & $7.52 \mathrm{mK}^{\dagger}$ & $14.43 \mathrm{~K}^{\dagger}$ \\
\hline
\end{tabular}

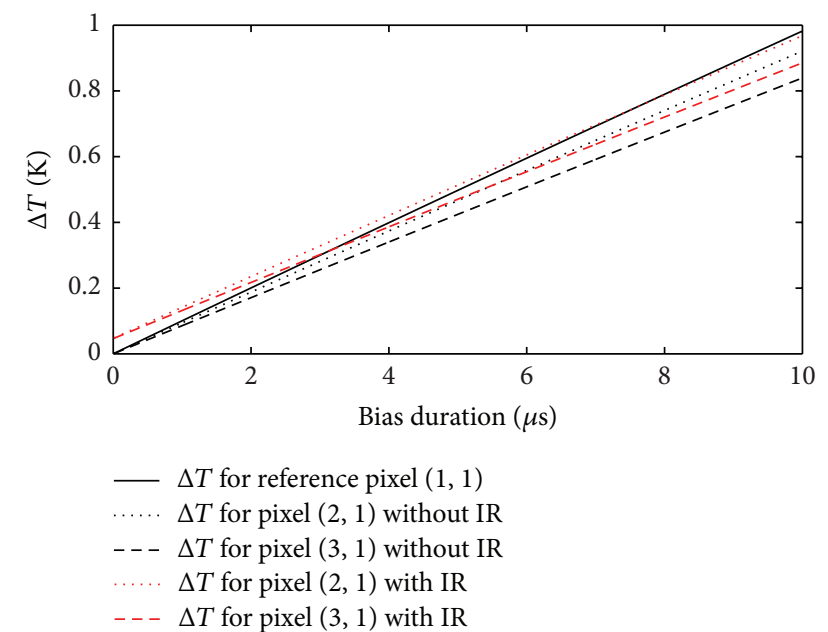

FIGURE 6: Temperature variation of the microbolometer with and without infrared radiation.

of the thermal isolation structure. The worst-case scenario is when the conductivity of the reference microbolometer is the same as that of the normal microbolometer. In [26], an optimum reference detector design with thermal conductance of $50 \mathrm{nW} / \mathrm{K}$ for uncooled resistive microbolometer is presented.

Figure 6 simulates the temperature response of microbolometers with and without infrared radiation. The microbolometers were biased with a bias current of $10 \mu \mathrm{A}$ for a duration of $10 \mu \mathrm{s}$. It can be seen from the figure that when the microbolometers were not exposed to infrared radiation, their temperature changed linearly during the readout time and there was no DC offset before the start of the readout. Similarly, when the microbolometers were exposed to infrared radiation, there was a temperature drift due to the incoming radiant flux. It was assumed that all the microbolometers had achieved saturation of the temperature difference before the start of the readout; that is, the readout of the microbolometers would start after $50 \mathrm{~ms}$ of exposing the microbolometers to infrared radiation. The temperature rise due to the incident infrared power was reflected by the small temperature step at the start of the readout. The temperature change due to infrared power was very small as compared to the temperature rise due to the self-heating.

2.4. Thermal Imager Simulator. When microbolometers are fabricated, they suffer from process variability that introduces an FPN into the thermal image. For the simulation purpose, a process variation of $\pm 10 \%$ is considered and a uniformly distributed noise is introduced in the $100 \mathrm{k} \Omega$ nominal resistance that serves as an FPN. Algorithm 1 shows the algorithm for the thermal imager simulator that works in two steps. The first step is the calibration mode and the second step is the image acquisition mode. In the calibration mode, the dark condition is set to true. The infrared power is set to zero so that there is no change in the resistance of microbolometer due to the incident radiation. The calibration data are acquired by covering the lens of the thermal camera, that is, by not exposing the microbolometers to infrared power. The thermal parameters of the microbolometer along 


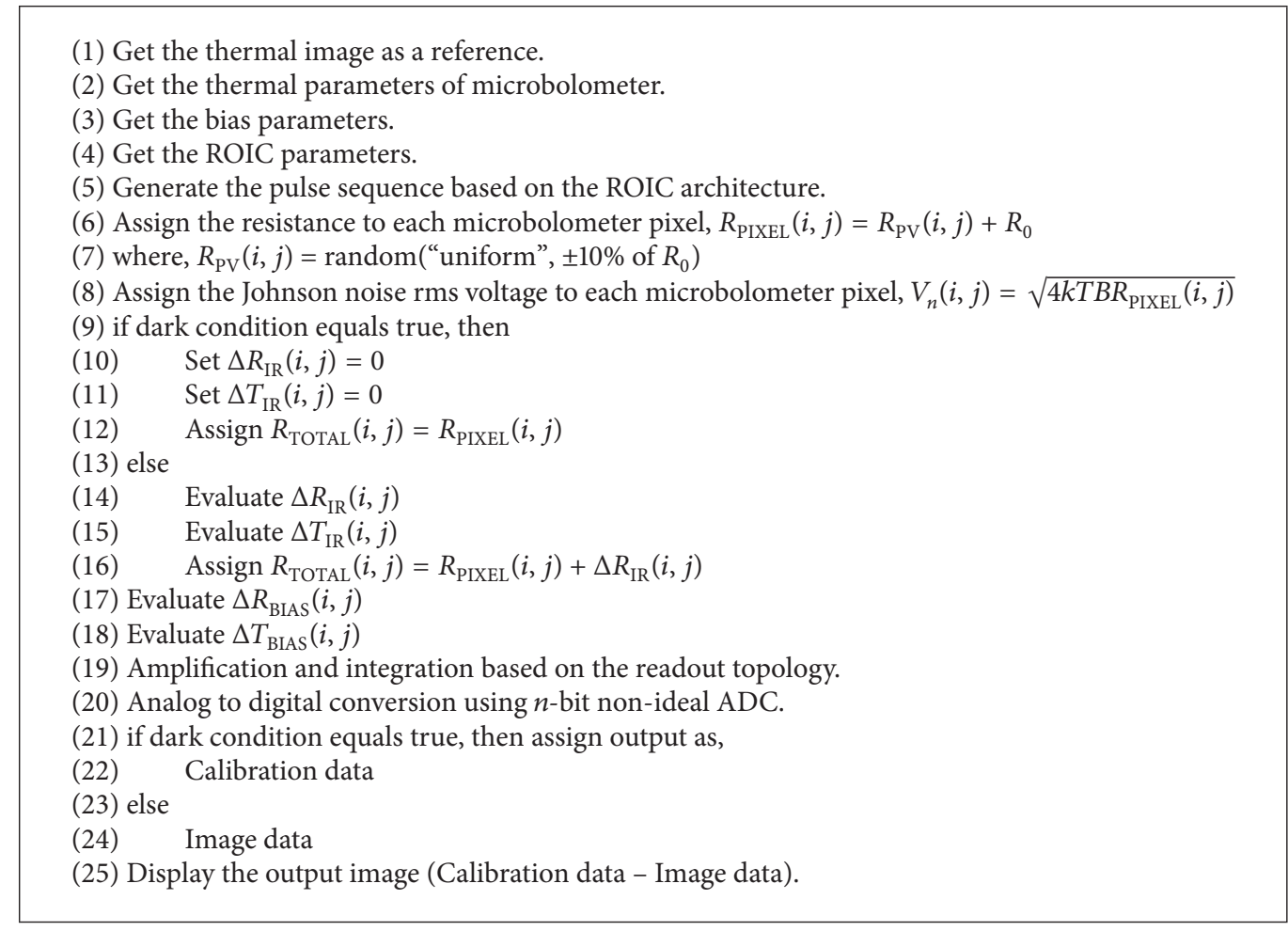

Algorithm 1: Algorithm for the thermal imager simulator.

TABLE 5: Test cases for reference based serial readout and column-wise readout.

\begin{tabular}{|c|c|c|c|c|c|c|}
\hline \multirow{2}{*}{ Cases } & \multirow{2}{*}{$\begin{array}{l}\text { Thermal conductance of the normal } \\
\text { microbolometer }\end{array}$} & \multirow{2}{*}{$\begin{array}{l}\text { Thermal conductance of the reference } \\
\text { microbolometer }\end{array}$} & \multicolumn{4}{|c|}{ Simulation parameters } \\
\hline & & & $I_{\mathrm{BIAS}}(\mu \mathrm{A})$ & $T_{\text {BIAS }}(\mu \mathrm{s})$ & ADC (bits) & $V_{\text {MAX,MIN }}(\mathrm{mV})$ \\
\hline Case I & $3.40 e-8$ & $9.50 e-9$ & 10 & 6 & 14 & \pm 600 \\
\hline Case II & $3.40 e-8$ & $9.50 e-9$ & 20 & 6 & 14 & \pm 600 \\
\hline Case III & $3.40 e-8$ & $1.44 e-7$ & 20 & 6 & 14 & \pm 600 \\
\hline Case IV & $3.40 e-8$ & $3.40 e-8$ & 15 & 6 & 14 & \pm 600 \\
\hline Case V & $3.40 e-8$ & $1.44 e-7$ & 30 & 6 & 14 & \pm 600 \\
\hline
\end{tabular}

with the bias and ROIC parameters are provided to the simulator. A pulse sequence is generated for the readout of the microbolometers based on the pulse sequence provided in Table 3. The complete readout is performed and the data acquired at this stage serves as the calibration data. The calibration operation does not need to be performed every time a new thermal image is taken.

The next step is to acquire the thermal image. In the image acquisition mode, the dark condition is set to false. A grayscale thermal image is provided as an input to the simulator. The focal plane array is exposed to the infrared power for few $\mathrm{ms}$ so that all the normal microbolometers achieve the saturation of temperature difference before the readout start. The incoming radiant flux only changes the resistance of normal microbolometers since the reference is an infrared blind microbolometer whose resistance only changes due to the electrical biasing. The complete readout is performed again based on the pulse sequence provided in Table 3 and the acquired data serves as the image data.
The resistance of reference microbolometer only changes due to the self-heating, in both the calibration mode and the image acquisition mode. But the resistance of normal microbolometer changes due to the self-heating in the calibration mode and self-heating along with infrared power in the image acquisition mode. The final thermal image is produced by subtracting the calibration data from the image data. A differential amplifier is used to remove the common mode signals and to perform the amplification of the readout voltage. A 14-bit nonideal ADC is used to perform the analogto-digital conversion, and the resulting digital information is used to display an output thermal image.

\section{Results}

Table 5 shows the test cases that were used to simulate the serial readout architecture using the proposed simulator. The serial readout topology was a pair-wise timemultiplexed row-wise readout architecture in which one pair 


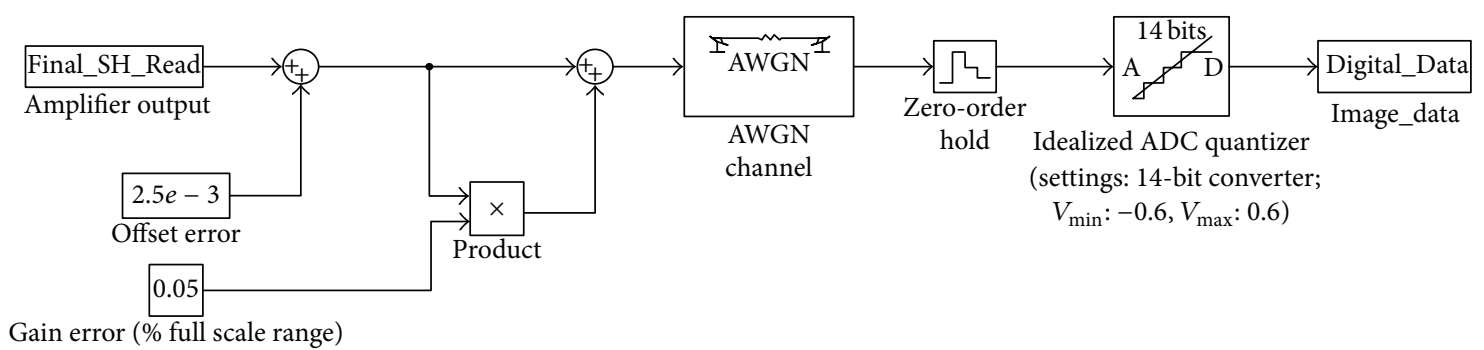

FIGURE 7: Nonideal ADC with offset error, gain error, and white Gaussian noise.

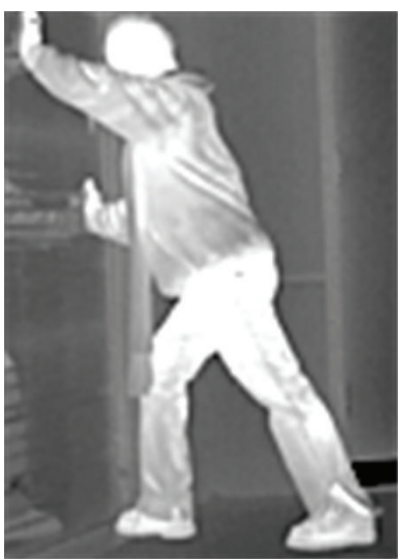

(a)

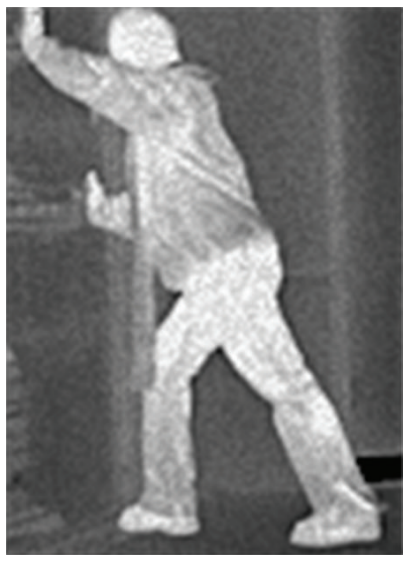

(e)

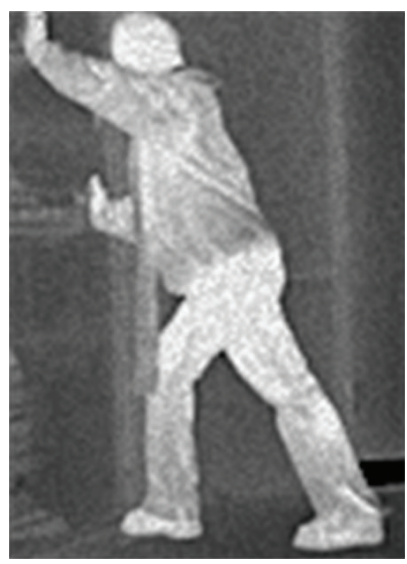

(b)

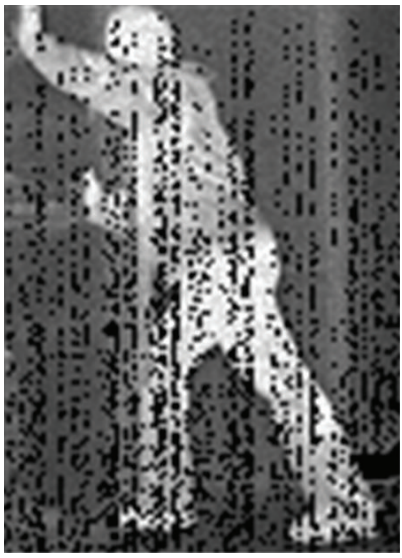

(f)

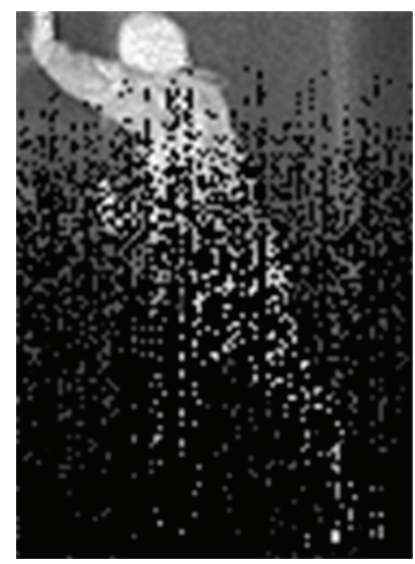

(c)

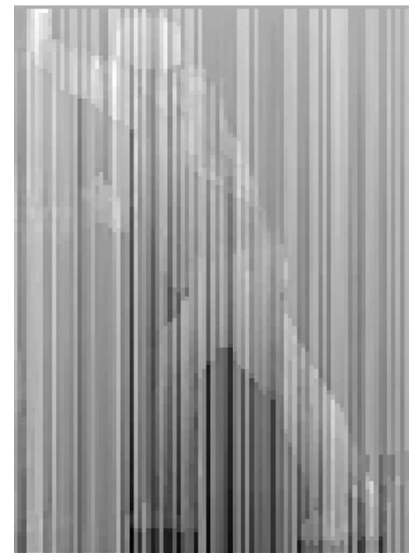

(g)

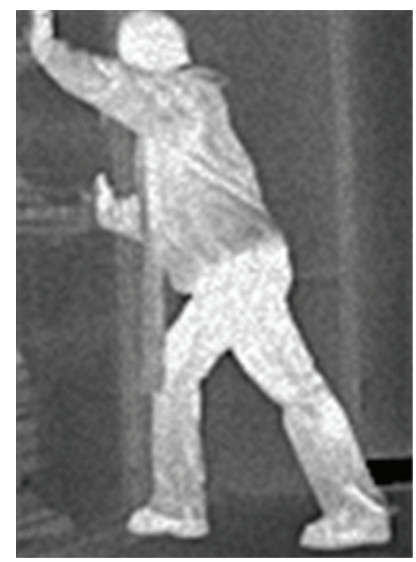

(d)

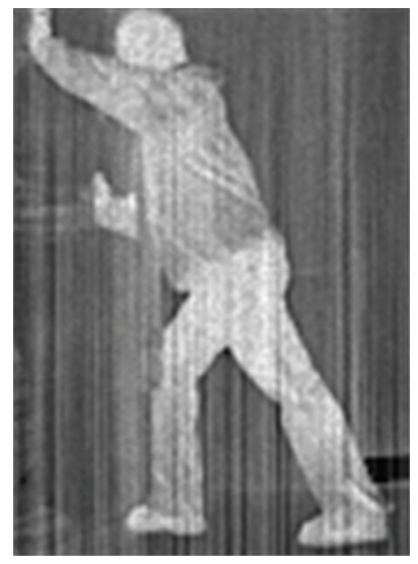

(h)

Figure 8: (a) Input thermal image, (b) output for the test cases mentioned in Table 3, case I, (c) case II, (d) case III, (e) case IV, (f) case V, (g) output for $I_{\text {BIAS }}=20 \mu \mathrm{A}, T_{\text {BIAS }}=250 \mu$ s using column-wise readout, and (h) output for case IV mentioned in Table 3 using pair-wise serial readout.

of microbolometers was biased and readout was performed. The normal and reference microbolometers were biased such that they followed the temperature variation shown in Figures 3 (a) and 3(b), respectively.

To be realistic, the Johnson noise was included in the modeling of uncooled microbolometer. The output of the differential amplifier was added with an offset error, gain error, and white Gaussian noise to model the nonideal ADC as shown in Figure 7.
Figure 8(a) shows the input thermal image to the simulator. For the simulation purpose, it was assumed that all the microbolometers were exposed to the infrared input power long enough to achieve saturation of the temperature difference. For case I and case II, the thermal conductance of the reference microbolometer was kept lower than that of the normal microbolometer. Figure $8(\mathrm{~b})$ is an image output of the simulator when the bias current was set to $10 \mu \mathrm{A}$ for a duration of $6 \mu \mathrm{s}$. For the serial readout architecture with a 


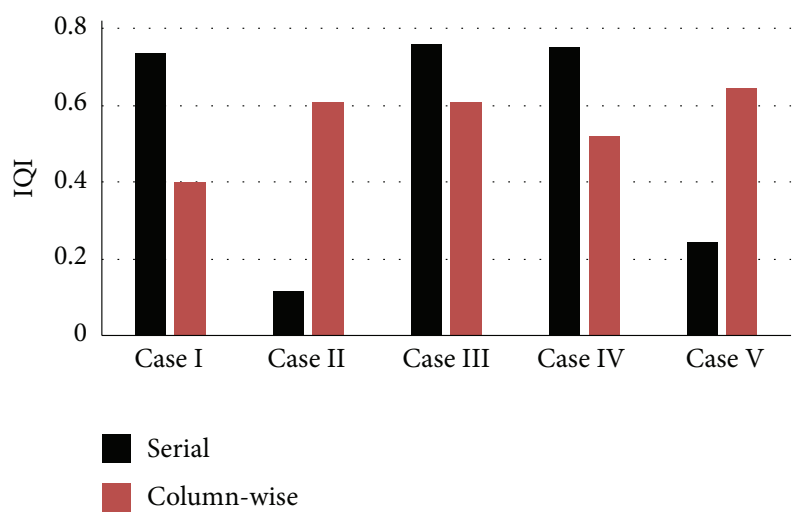

FIGURE 9: Image quality index for the test cases mentioned in Table 3.

reference pair that was biased multiple times within the frame period, such low value of the bias current is acceptable.

For case II when the bias current was $20 \mu \mathrm{A}$, most of the pixels were black, as shown in Figure 8(c). The reason is that when the bias current was large, the temperature of the microbolometer rises to a significantly high value due to the self-heating. Since resistive microbolometers have a negative temperature coefficient of resistance, the rise in temperature decreases their resistance, which in turn decreases the voltage drop across the reference microbolometer. The decrease in voltage drop results in higher output of the differential amplifier. If the output of the differential amplifier is higher than the maximum voltage swing of the ADC, then the output of the ADC for that particular pixel gets saturated and appears black. In case III, the bias current was kept the same as that in case II, but the thermal conductance of the reference microbolometer was larger than that of the normal microbolometer. The impact of self-heating was drastically reduced since the reference microbolometer cooled down very sharply before it was read out with the next row owing to its low thermal time constant. Figure 8(e) shows the worstcase scenario for a reference microbolometer, when the reference and normal microbolometers have the same thermal conductance. The bias current was slightly reduced for case IV to lower the self-heating. In case V, the bias current was further increased to $30 \mu \mathrm{A}$ for the reference microbolometer with high thermal conductance, which resulted in a distorted image because of very high self-heating. Figure $8(\mathrm{~g})$ shows the output of the thermal imager simulator for $I_{\text {BIAS }}=$ $20 \mu \mathrm{A}, T_{\mathrm{BIAS}}=250 \mu \mathrm{s}$ using column-wise readout. When the microbolometers are biased for a longer duration, then the temperature drift due to the self-heating is significantly large. It will result in the introduction of vertical strip noise in the thermal image as seen in Figure 8(g). Different readout topologies require different bias parameters, depending on the amplification and integration methodology.

Image quality index (IQI) is an image distortion assessment technique that was used as a statistical tool to compare the results of the test cases mentioned above. IQI considers three major factors under consideration while evaluating the image distortion: loss of correlation, luminance distortion, and contrast distortion [27]. The dynamic range of IQI is $[-1,1]$. In order to have a strong relationship between an input thermal imager and output image, the IQI should approach 1 . Test cases mentioned in Table 5 were simulated for the reference-based serial readout and column-wise readout using thermal imager simulator. The frame time for a serial readout architecture was set to $100 \mathrm{~ms}$ whereas for a column-wise readout it was set to $10 \mathrm{~ms}$. The quantitative comparative analysis between the two architectures is shown in Figure 9.

It can be inferred from the results that, except for case II and case V, the reference-based serial readout has better IQI than the column-wise readout. In case II, the thermal conductivity of the reference microbolometer is very small as compared to that of the normal microbolometer, whereas, in case V, the bias current is too large, thus introducing large temperature drift due to the self-heating. In order to improve IQI for the reference-based serial readout, the bias current should be kept within the region of interest, as shown in Figure 2. Similarly, if the thermal conductance of the reference microbolometer is higher than that of the normal microbolometer, then, for a higher value of bias current, a better thermal image with low noise can be achieved. Further increasing the bias current introduces more noise in the image. On the other hand, IQI for the column-wise readout architecture does not significantly vary based on the thermal conductance of the reference microbolometer. Since all the microbolometers including the reference microbolometer were biased only once during the entire frame period, the reference-based serial readout was preferred over the column-wise one because the power dissipation was very low and, for the optimum value of the bias current, better IQI was achieved.

In order to make a comparison between reference-pair serial and pair-wise serial readout, case IV of Table 5 was simulated for pair-wise serial readout. Figure 8(h) shows the output for case IV using pair-wise serial readout architecture. The IQI for case IV for reference-pair serial is 0.75 whereas for pair-wise serial the IQI is 0.51 . The power dissipation for both serial readout architectures is approximately the same, because the pair-wise serial architecture uses the same resources as those of the reference-pair serial readout, that is, one amplifier and one ADC, and selects a pair of microbolometers at a time but differs in terms of microbolometer selection and readout. The reference-pair serial readout puts strict requirements on the dynamic range of the readout as compared to the pair-wise serial but, in terms of image quality, the reference-pair serial readout shows better results as compared to the pair-wise serial for optimum bias parameters.

\section{Conclusion}

In this paper, a detailed thermal behavior and theoretical analysis of uncooled microbolometer was presented. The impact of self-heating on the performance of the thermal imager was explored. Choosing an appropriate bias parameter is one of the most important tasks while designing an ROIC. The bias current should be such that it does not 
compromise the responsivity of the microbolometer and, at the same time, does not increase the temperature of the microbolometer membrane owing to the self-heating. Similarly, the bias duration should be very small as compared to the thermal time constant of the microbolometer; otherwise, it will result in a large temperature drift owing to the selfheating. Moreover, it can be inferred from the results that the temperature rise due to bias power was much higher than that due to incident power. A thermal imager simulator was presented which was used as a tool to visually analyze the impact of noise on the quality of the thermal image. The proposed simulator can serve as a useful tool for the designers to find an optimum value of the bias parameters taking into account the acceptable temperature rise due to the self-heating. In this work, a serial readout architecture was explored with a blind microbolometer as a reference. The quantitative comparative analysis between the serial and column-wise readout was presented. The serial readout was preferred because of low power dissipation and better IQI.

\section{Competing Interests}

The authors declare that they have no competing interests.

\section{Acknowledgments}

This work was supported by the Research Center of College of Computer and Information Sciences, King Saud University. The authors are grateful for this support.

\section{References}

[1] B. Mesgarzadeh, M. R. Sadeghifar, P. Fredriksson, C. Jansson, F. Niklaus, and A. Alvandpour, "A low-noise readout circuit in 0.35- $\mu \mathrm{m}$ CMOS for low-cost uncooled FPA infrared network camera," in Infrared Technology and Applications XXXV, vol. 7298 of Proceedings of SPIE, pp. 1-8, 2009.

[2] M. Perenzoni, D. Mosconi, and D. Stoppa, "A $160 \times 120$-pixel uncooled IR-FPA readout integrated circuit with on-chip nonuniformity compensation," in Proceedings of the ESSCIRC, pp. 122-125, Seville, Spain, September 2010.

[3] P. Neuzil and T. Mei, "A method of suppressing self-heating signal of bolometers," IEEE Sensors Journal, vol. 4, no. 2, pp. 207-210, 2004.

[4] D. A. Ramirez, Focal Plane Arrays (FPAs) Operation Principles, 2006.

[5] D. Svärd, C. Jansson, and A. Alvandpour, "A readout IC for an uncooled microbolometer infrared FPA with on-chip selfheating compensation in $0.35 \mu \mathrm{m}$ CMOS," Analog Integrated Circuits and Signal Processing, vol. 77, no. 1, pp. 29-44, 2013.

[6] R. K. Bhan, R. S. Saxena, C. R. Jalwania, and S. K. Lomash, "Uncooled infrared microbolometer arrays and their characterisation techniques," Defence Science Journal, vol. 59, no. 6, pp. 580-589, 2009.

[7] S. B. Ju, Y. J. Yong, and S. G. Kim, "Design and fabrication of a high fill factor microbolometer using double sacrificial layers," in Proceedings of the Infrared Technology and Applications XXV, vol. 3698 of Proceedings of SPIE, pp. 1-10, Orlando, Fla, USA, July 1999.

[8] F. Niklaus, C. Vieider, and H. Jakobsen, "MEMS-based uncooled infrared bolometer arrays: a review," in Proceedings of the MEMS/MOEMS Technologies and Applications III, vol. 6836, Beijing, China, November 2007.

[9] S. J. Hwang, H. H. Shin, and M. Y. Sung, "High performance read-out IC design for IR image sensor applications," Analog Integrated Circuits and Signal Processing, vol. 64, no. 2, pp. 147$152,2010$.

[10] P. J. Thomas, A. Savchenko, P. M. Sinclair et al., "Offset and gain compensation in an integrated bolometer array," in Infrared Technology and Applications XXV, vol. 3698 of Proceedings of SPIE, pp. 826-836, 1999.

[11] M. Krupiński, G. Bieszczad, T. Sosnowski, H. Madura, and S. Gogler, "Non-uniformity correction in microbolometer array with temperature influence compensation," Metrology and Measurement Systems, vol. 21, no. 4, pp. 709-718, 2014.

[12] R. M. Hussey and W. H. Havens, "Fixed Pattern Noise Compensation Method and Apparatus," US 20080143855 A1, Jun 19, 2008.

[13] G. Bieszczad, T. Orżanowski, T. Sosnowski, and M. Kastek, "Method of detectors offset correction in thermovision camera with uncooled microbolometric focal plane array," in ElectroOptical and Infrared Systems: Technology and Applications VI, vol. 7481 of Proceedings of SPIE, 2009.

[14] H. H. Shin, S. J. Hwang, E. S. Jung, S. W. Yu, and M. Y. Sung, "A readout IC design for the FPN reduction of the bolometer in an IR image sensor," Transactions on Electrical and Electronic Materials, vol. 8, no. 5, pp. 196-200, 2007.

[15] W. J. Parrish and J. T. Woolaway II, "Improvements in uncooled systems using bias equalization," in Infrared Technology and Applications XXV, vol. 3698 of Proceedings of SPIE, pp. 748-755, 1999.

[16] S. I. Haider, S. Majzoub, M. Alturaigi, and M. Abdel-Rahman, "Pair-wise serial ROIC for uncooled microbolometer array," IEIE Transactions on Smart Processing and Computing, vol. 4, no. 4, pp. 251-257, 2015.

[17] D. Jakonis, C. Svensson, and C. Jansson, "Readout architectures for uncooled IR detector arrays," Sensors and Actuators A: Physical, vol. 84, no. 3, pp. 220-229, 2000.

[18] X. Gu, G. Karunasiri, J. Yu, G. Chen, U. Sridhar, and W. J. Zeng, "On-chip compensation of self-heating effects in microbolometer infrared detector arrays," Sensors and Actuators A: Physical, vol. 69, no. 1, pp. 92-96, 1998.

[19] M. V. S. Ramakrishna, G. Karunasiri, P. Neuzil, U. Sridhar, and W. J. Zeng, "Highly sensitive infrared temperature sensor using self-heating compensated microbolometers," Sensors and Actuators A: Physical, vol. 79, no. 2, pp. 122-127, 2000.

[20] S. I. Haider, S. Majzoub, M. Alturaigi, and M. Abdel-Rahman, "Column-wise ROIC design for uncooled microbolometer array," in Proceedings of the 1st International Conference on Information and Communication Technology Research (ICTRC '15), pp. 56-59, May 2015.

[21] B. Hu, P. Li, A.-W. Ruan, and D.-M. Lin, "Design and verification of $160 \times 120$ bolometer ROIC," in Proceedings of the 2011 International Conference on Computational Problem-Solving (ICCP '11), pp. 275-278, IEEE, Chengdu, China, October 2011.

[22] R. D. Gow, D. Renshaw, K. Findlater et al., "A comprehensive tool for modeling CMOS image-sensor-noise performance," IEEE Transactions on Electron Devices, vol. 54, no. 6, pp. 13211329, 2007.

[23] G. Bieszczad and M. Kastek, "Measurement of thermal behavior of detector array surface with the use of microscopic thermal camera," Metrology and Measurement Systems, vol. 18, no. 4, pp. 679-690, 2011. 
[24] L. L. Noc, O. Pancrati, M. Doucet, D. Dufour, and B. Debaque, "Software thermal imager simulator," in Proceedings of the Electro-Optical and Infrared Systems: Technology and Applications XI, vol. 9249 of Proceedings of SPIE, Amsterdam, The Netherlands, September 2014.

[25] K. Chrzanowski and M. Krpuski, "Computer simulator for training operators of thermal cameras," in Enhanced and Synthetic Vision 2004, 187, vol. 5424 of Proceedings of SPIE, August 2004.

[26] M. Tepegoz, F. Civitci, and T. Akin, "An optimum reference detector design for uncooled microbolometer FPAs," in Proceedings of the Infrared Technology and Applications XXXIV, vol. 6940 of Proceedings of SPIE, Orlando, Fla, USA, March 2008.

[27] Z. Wang and A. C. Bovik, "A universal image quality index," IEEE Signal Processing Letters, vol. 9, no. 3, pp. 81-84, 2002. 


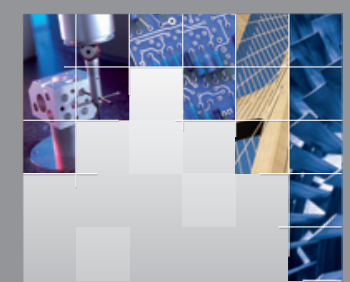

\section{Enfincering}
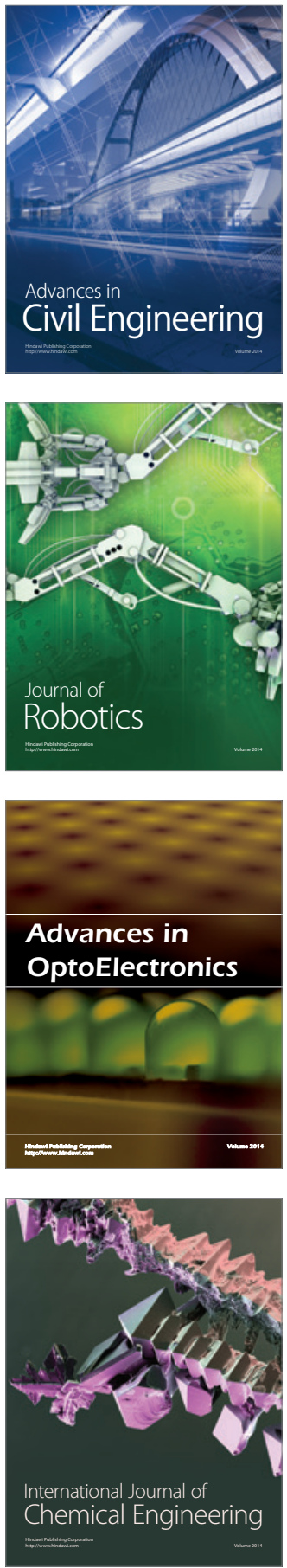

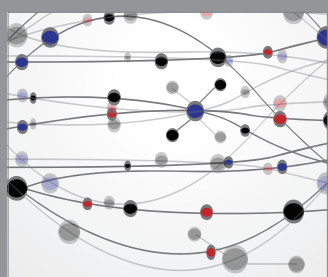

The Scientific World Journal

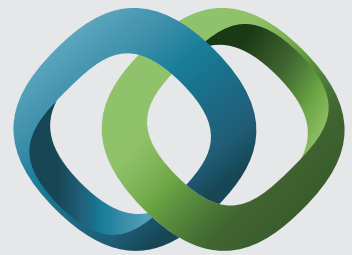

\section{Hindawi}

Submit your manuscripts at

http://www.hindawi.com
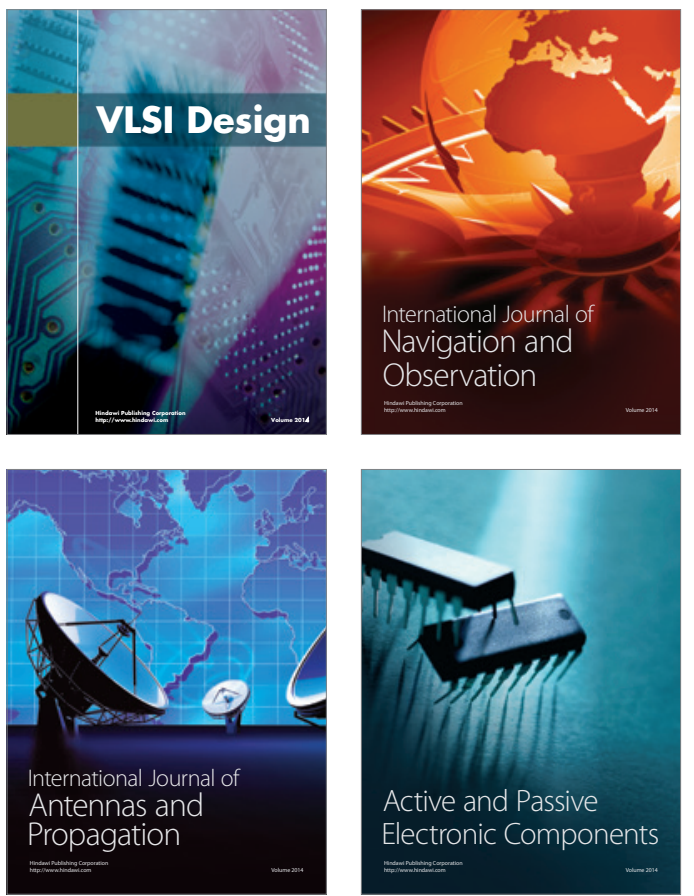
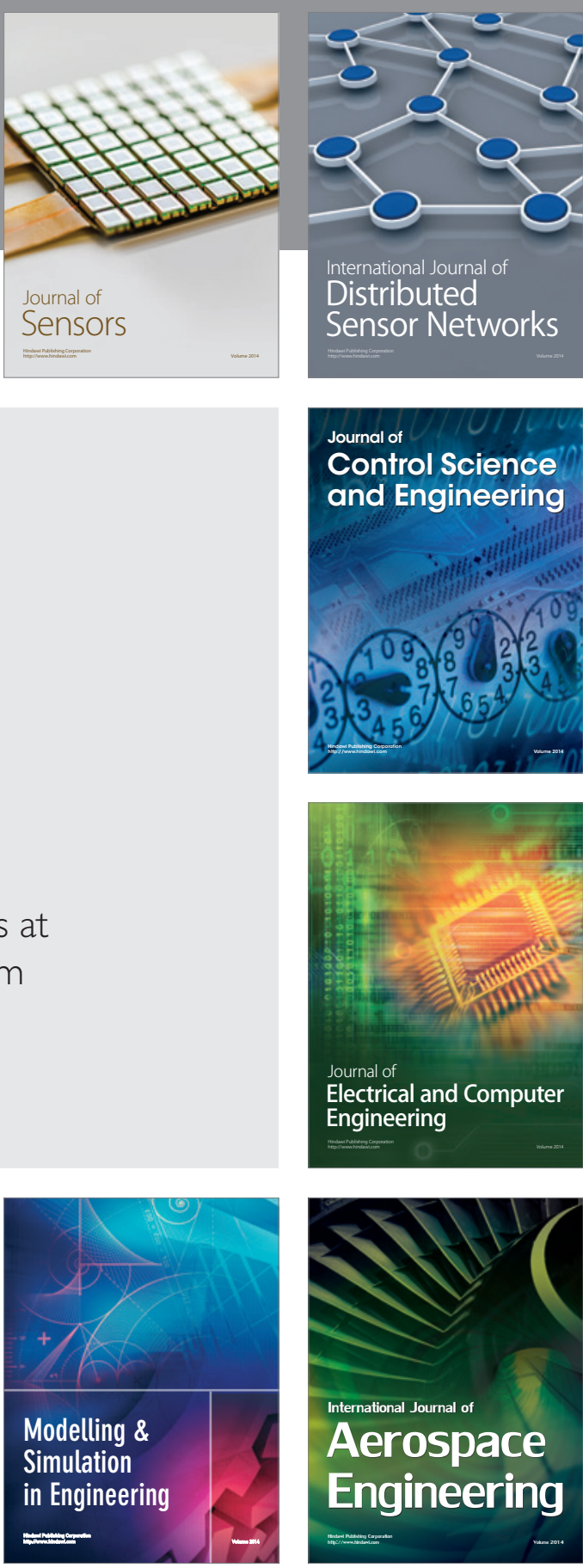

International Journal of

Distributed

Sensor Networks

Journal of

Control Science

and Engineering
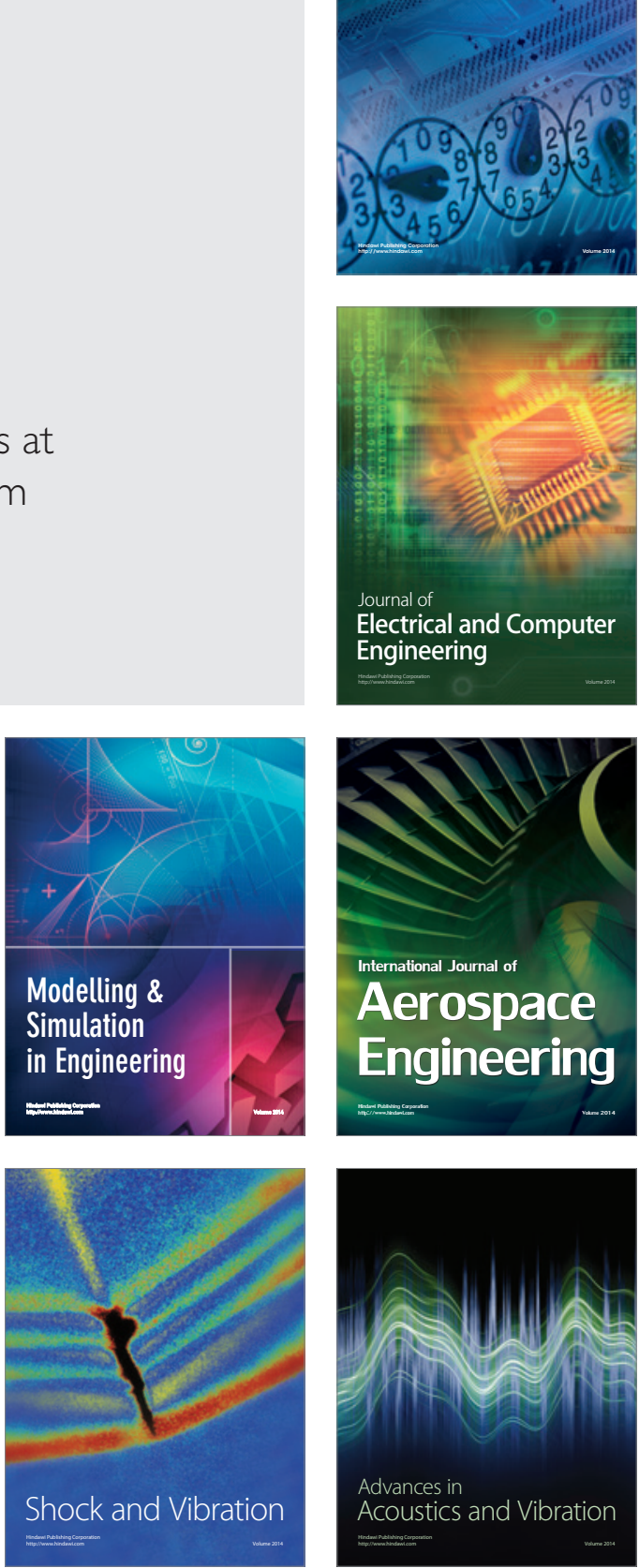